\title{
Finite element analysis of a crack tip in silicate glass: No evidence for a plastic zone
}

\author{
T. Fett,,${ }^{1,2}$ G. Rizzi, ${ }^{1}$ D. Creek, ${ }^{2}$ S. Wagner, ${ }^{2}$ J. P. Guin, ${ }^{3}$ J. M. López-Cepero, ${ }^{4}$ and S. M. Wiederhorn ${ }^{5}$ \\ ${ }^{1}$ Forschungszentrum Karlsruhe, Institut für Materialforschung II, D-76021 Karlsruhe, Germany \\ ${ }^{2}$ Institut für Keramik im Maschinenbau, Universität Karlsruhe, D-76131 Karlsruhe, Germany \\ ${ }^{3}$ LARMAUR FRE-CNRS 2717, University of Rennes I, Rennes, France \\ ${ }^{4}$ Department of Condensed Matter Physics, University of Seville, Seville, Spain \\ ${ }^{5}$ National Institute of Standards and Technology, Gaithersburg, Maryland 20899-8520, USA \\ (Received 8 February 2007; revised manuscript received 30 October 2007; published 15 May 2008)
}

\begin{abstract}
Recently, the claim was made that cracks in silicate glasses propagate by the nucleation, growth, and coalescence of cavities at crack tips, which is the same way as in metals but at a much smaller scale. This hypothesis for crack growth is based in part on the measurement of surface displacements near the tip of an emerging crack, which is the point at which a crack front intersects the side surface of the specimen. Surface displacements measured by atomic force microscopy were less than theoretically predicted. The difference between the theoretical and experimental displacements was attributed to a plastic zone surrounding the tip of the moving crack. In this paper, we show that the theoretical analysis used earlier was based on an incorrect assumption about the functional dependence of the displacement with distance from the crack tip. We use a full three-dimensional finite element analysis combined with an asymptotic solution of the crack geometry to obtain a solution to the surface displacement problem. We show that the calculated displacements are fully consistent with those experimentally measured by using an atomic force microscope. No divergence from elastic behavior is observed. Our results support the view that crack propagation in glass is entirely brittle. No evidence for plasticity at the crack tips is obtained.
\end{abstract}

DOI: 10.1103/PhysRevB.77.174110

PACS number(s): 62.20.M-, 61.43.Fs

\section{INTRODUCTION}

In several recent papers, experiments were carried out on silicate glasses to monitor surface displacements in the vicinity of crack tips. Because of its high resolution, the atomic force microscope (AFM) is ideal for this purpose. Two kinds of experiments were carried out. In one, crack-tip opening displacements were measured at some small distance from the crack tip $;^{1,2}$ in the second, displacements were measured normal to specimen surfaces near the point of intersection of the crack front with the side surface of the specimen. ${ }^{3}$ From these measurements, the investigators concluded that surface displacements near the crack tip were not consistent with those expected if the deformation was entirely elastic. They attributed this discrepancy to the existence of a zone of inelastic or plastic deformation in the vicinity of the crack tip. ${ }^{1-3}$

The authors of Ref. 3 also reported cavity formation at the tips of propagating cracks and claimed that the main mechanism of crack growth in silicate glasses was by the nucleation, growth, and coalescence of cavities at crack tips in a glass. Data supporting this view were obtained by observing the tip of a moving crack with an AFM. The data collected suggested that the size of the cavities in a lithium aluminosilicate glass was about $20 \mathrm{~nm}$ in length and $5 \mathrm{~nm}$ in height. The depth of the cavities could not be determined with the AFM.

The conclusion that inelastic or plastic process zones surround crack tips in a glass is in conflict with earlier theoretical and experimental results on the nature of deformation near crack tips in glasses and other brittle ceramics. ${ }^{4,5}$ By using transmission electron microscopy, investigators were able to show that cracks in sapphire, silicon, germanium, silicon carbide, and $\mathrm{SiAlON}$ propagated at room temperature without the generation and movement of dislocations from the crack tips. ${ }^{4,6-10}$ When some of these materials (sapphire, silicon) were fractured at high temperatures, dislocations were easily observed at the crack tips, suggesting that if the dislocations were present at room temperature, they, too, would have been easily seen. Silicate glasses do not deform by dislocation motion, but because the type of bonding in silicate glasses is similar to that in the materials examined in Refs. 4 and 6-10. The authors of Ref. 4 concluded that the fracture of silicate glasses also occurs without the intervention of plastic deformation.

In the present paper, we address the problem of plastic deformation near crack tips in silicate glasses. In particular, we examine the magnitude of specimen surface displacement $u_{z}$, near the tip of an emerging crack, i.e., the point at which the crack front intersects the side surface of the specimen. Displacements obtained through a three-dimensional finite element analysis are compared to experimentally determined displacements measured by atomic force microscopy. ${ }^{3}$ Our finite element analyses are consistent with those displacement measurements. ${ }^{3}$ In particular, the displacement is finite at the crack tip, which is in contrast to a theoretical solution given in Ref. 3. The agreement between the finite element calculation and the AFM measurements suggests that the crack-tip displacements in the glass used in Ref. 3 can be rationalized in terms of elastic processes near the crack tip. There is no need to invoke the idea of nanoscale plasticity to explain the results.

We do not address the question of cavity formation in this paper. This topic has been addressed in other publications, ${ }^{11-13}$ wherein AFM data on fracture surfaces were presented to illustrate that no cavities can be detected in silicate glasses. In this paper, we just deal with issues related 


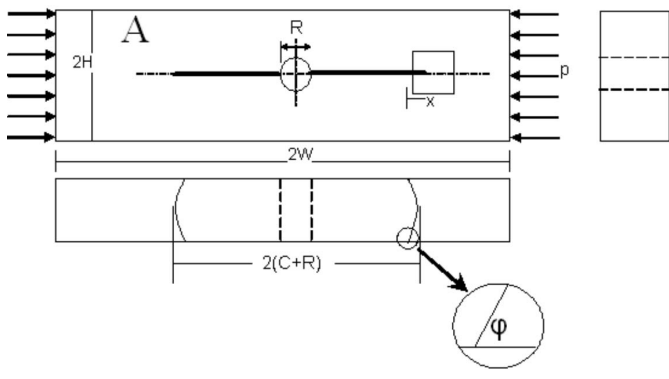

FIG. 1. The DCDC specimen. The square surrounding the righthand crack tip shows the area that was scanned with the atomic force microscope. The parameter $p$ is the compressive stress applied to the ends of the specimen. The crack surface is normal to surface $A$ and the crack front makes an angle $\varphi$ to surface $A$. The displacement $u_{z}$ is measured at the crack tip on surface $A$ (within the square at the crack tip).

to the elastic solution of the surface displacement near an emerging crack and its relationship to the possibility of plastic deformation at the crack tips in a glass. We will show that the conclusion made in Ref. 3 that plastic deformation occurs at the crack tips in a glass is a consequence of a mistaken assumption about the dependence of surface displacement $u_{z}$ on distance $r$ from the crack tip. With the correct functionality, no divergence from elastic behavior is observed.

\section{REVIEW OF EARLIER WORK ON DEPRESSIONS NEAR CRACK TIPS}

Célarié $e t ~ a l .{ }^{3}$ measured the height profile of the free surface near the tip of a growing crack by using an atomic force microscope. The glass was a lithium aluminosilicate glass with unspecified composition and manufacturer. Doublecleavage drilled-compression specimens ${ }^{14-16}$ (DCDC) (Fig. 1) were used in these studies because they provided a stable platform for measuring crack-tip profiles by AFM. Measurements were made on surface $A$ (Fig. 1) within the square surrounding the crack tip.

Profile measurements near the crack front emerging from surface $A$ of the DCDC specimen resulted in the topography shown in Fig. 2. A height profile along the $x$ axis (along the crack plane) can be seen in Fig. 2(a); a profile along the $y$ axis is shown in Fig. 2(b). The crack-tip position, $x_{0}$ and $y_{0}$ in Fig. 2, is identified by the vertical dashed-dotted lines.

The authors of Ref. 3 derived a relationship for surface displacements $u_{z}$ lying normal to surface $A$ in Fig. 1:
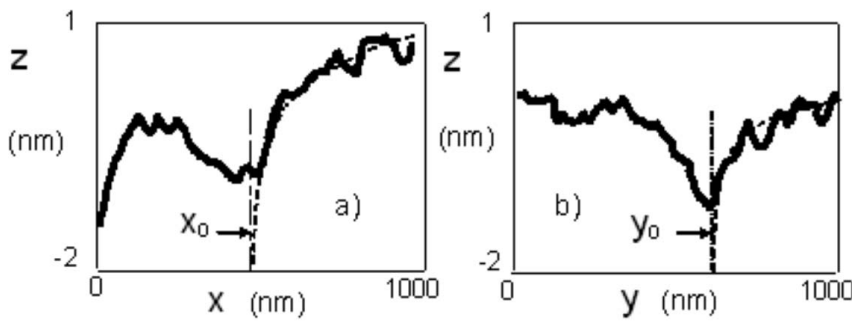

FIG. 2. Height profile measured around a crack tip (Ref. 3).
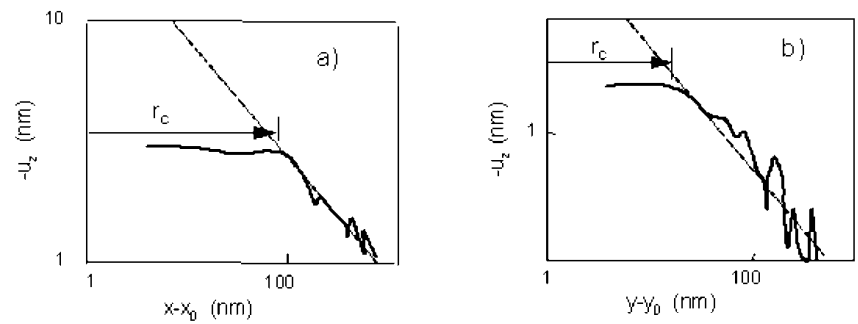

FIG. 3. Comparison of measured displacements $u_{z}$ with calculated displacements, $u_{z}=\boldsymbol{B} / \sqrt{r}$, where $B$ is a constant of the fit. Logarithmic curves of $u_{z}$ are plotted as a function of the logarithm of the distance from the crack tip. The measured displacements are given by the solid curves; the calculated displacements are given by the dashed straight lines. At distances from the crack tip greater than a critical value $r_{c}$, the measured and calculated displacements overlap. (a) Displacements along $y=0$; (b) displacements along $x$ $=0$.

$$
u_{z}=z-z_{o}=-2 \cos (\theta / 2) B \nu K_{I} /(E \sqrt{2 \pi r}),
$$

where $r$ and $\theta$ are polar coordinates originating at the crack tip, $E$ is Young's modulus, $v$ is Poisson's ratio, and $B$ is the thickness of the specimen. These parameters have wellknown values in Eq. (1) and are not determined by fitting procedures. The parameter $z_{o}$ is the position of the surface.

Equation (1) was not used for any of the discussions in Ref. 3 Instead, the authors assumed that the displacement had a $1 / \sqrt{r}$ dependency and fitted an equation with this dependency, $u_{z}=-B / \sqrt{r}$, to the data shown in Fig. 2. The parameter $B$ bears no relationship to the coefficient of $1 / \sqrt{r}$ in Eq. (1). It is purely an empirical constant of the fit.

The empirical curve is given by the dashed line for $x$ $>x_{o}$ and $y>y_{o}$, where $x_{o}$ and $y_{o}$ are the zero points for the $x$ and $y$ axes, respectively. The curve matches the experimental data over a wide range of the data. However, in the region around the zero point, the empirical and experimental curves do not match. The differences between the measured displacements (solid curves) and the calculated displacements (dashed curves) in Fig. 2 were attributed to plastic deformation near the crack tip. ${ }^{3}$

Figure 3 gives a comparison of the measured displacements with the calculated displacements, $u_{z}=B / \sqrt{r}$, on a log$\log$ plot. The calculated displacements are obtained by fitting the equation to experimental data for $r>r_{c}$. The location $r_{c}$ at which deviations from the straight line occur is interpreted in Ref. 3 as the radius from the crack for plastic deformation. Depending on the polar angle $\theta$ from the crack tip, a plastic zone is believed to extend from 20 to $100 \mathrm{~nm}$ from the crack tip.

Before proceeding with our finite element analysis of the depression near an emerging crack, some additional comments on Eq. (1) are necessary. Equation (1), which was derived by using plane stress conditions, is not applicable to the problem it was intended to solve. The equation yields an infinitely negative displacement at the crack tip when this line is approached along $\theta=0$. However, if the crack tip is approached along $\theta=\pi$, Eq. (1) yields $u_{z}=0$. Thus, the compatibility condition is violated. Two extremely different dis- 
placements of the same point, $r=0$, are impossible because displacements must be continuous even in stress singularities. This is the meaning of the fundamental compatibility condition, which is valid for cracks under plane strain and plane stress conditions, which is in contrast to the evaluation in Ref. 3 (for this problem, see the review article by $\mathrm{Sih}^{17}$ ).

\section{ASYMPTOTIC SOLUTIONS OF CRACK FRONT INTERSECTIONS WITH A FREE SURFACE}

The deformation state at the surface of finite bodies is highly three dimensional, which means that a simple twodimensional plane stress approximation of the strain fields around the crack tip is bound to be incorrect in the vicinity of the crack tip. Descriptions of cracks intersecting free surfaces are well known in theoretical fracture mechanics for both straight and inclined crack fronts. Several studies deal with the asymptotic analyses that provide exact solutions of displacements for the vicinity of the crack-terminating point. ${ }^{18-23}$ From an asymptotic analysis, the near-tip sidesurface displacements $u_{z}$ depend on the distance $r$ from the crack tip by the following power law relationship:

$$
u_{z} \propto r^{\lambda}, \quad \lambda \geq 0 .
$$

Up to four singularity exponents $\left(\lambda_{1}, \lambda_{2}, \lambda_{3}, \lambda_{4}\right)$ can exist for cracks depending on the type of loading. We calculated $\lambda_{n}$ for all the cases of loaded cracks by using the techniques discussed in Refs. 21, 22, and 24 and found that $0 \leq \lambda_{n} \leq 1$. Thus, the power law exponent, $\lambda=-0.5$, used in Ref. 3 falls outside the range predicted by Eq. (2) and, consequently, is not consistent with earlier asymptotic analyses of this kind of problem.

To determine the terminating angle, DCDC tests on a soda lime silicate glass were performed. After propagating the crack by about $4 \mathrm{~mm}$, the specimens were unloaded and fractured by pressing a large sewing needle into the hole. The glass used was B270 from Schott, Mainz. The diameter of the sewing needle exceeded that of the hole by $10 \%$. The crack contour of the DCDC test was clearly visible under an optical microscope. Crack-terminating angles in the range of approximately $50^{\circ} \leq \varphi \leq 73^{\circ}$ were observed. Figure 4(a) shows a part of the crack front near the side surface. The crack-terminating angle is $\varphi=63^{\circ}$ in this case. The curved crack front is a consequence of a reduction in the stress intensity factor in the surface region [see Fig. 4(b)], resulting in reduced crack growth there. We used a terminating angle of $60^{\circ}$ in the finite element calculations given below. As will be discussed below, the surface displacements calculated by using this angle are consistent with the AFM displacements measured in Ref. 3.

The proportionality factor in Eq. (2) is governed by the special specimen geometry and loading conditions and cannot be obtained by an asymptotic analysis. Therefore, the proportionality factor has to be numerically determined. The exponent $\lambda$, by contrast, can be theoretically determined; $; 1,22$ it exclusively depends on the crack-terminating angle $\varphi$. To determine the proportionality factor, a three-dimensional finite element study was carried out. The finite element analysis was also used to determine the exponent $\lambda$. The mesh for
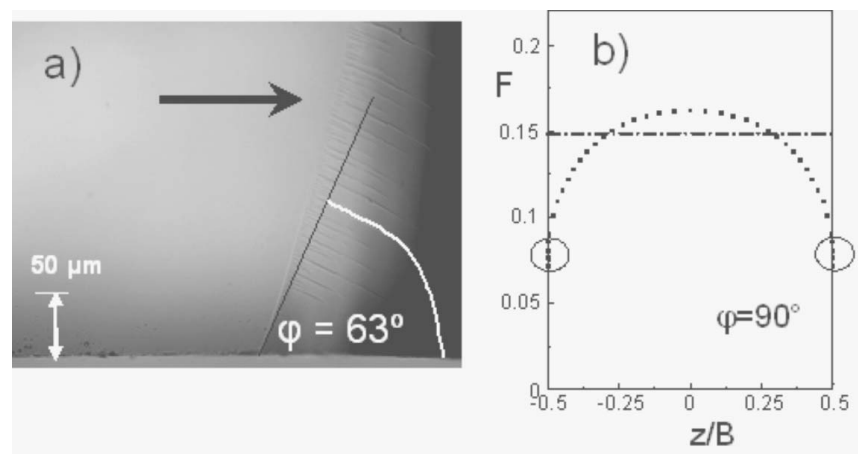

FIG. 4. (a) Crack contour at the free side surface, $y=0$. (b) Theoretical variation in the stress intensity factor (expressed by the geometric function $F \propto K$ ) along a straight crack, $\varphi=90^{\circ}$. At the surface (circles), the results are doubtful because of change in the singularity type of stresses.

the finite element analysis was fine enough, so that the value of $\lambda$ determined by the finite element analysis was equal to the theoretical value of $\lambda$ to within a small experimental scatter.

\section{THREE-DIMENSIONAL ELASTIC FINITE ELEMENT STUDY}

For the finite element computation, a crack in the DCDC specimen (Fig. 1) was modeled with $c / R=4, H / W=0.1$, and $R / H=0.25$. The specimen thickness $B$ was chosen as $B / W$ $=0.2$, resulting in a square cross section of the bar from which the specimen is made. We used 5600 elements with 27000 nodes to model $1 / 8$ of the specimen. The crack-tip region was modeled by collapsed two-dimensional elements. Computations were carried out with ABAQUS, Version 6.2, which provides the displacements $u_{z}$ as well as the individual stress intensity factors $K_{I}$ and $K_{I I}$.

In this context, it should be emphasized that it was not the aim of our analysis to model the displacement behavior at the crack-terminating point exactly. This seemed not possible to us because of the extremely large number of elements necessary. Several sizes and types of elements over the specimen thickness were chosen. To reach the asymptotic solution, we sequentially decreased the size of the near-tip mesh region until the displacement exponent $\lambda$ was close to the exact value obtained from asymptotic analyses. ${ }^{21,22}$ The element size continuously decreased while approaching the free surfaces. The two outer elements were drastically reduced to $1 / 100$ and $1 / 10$ of the smallest element of the bulk material. We assume that the material of analysis is homogeneous and isotropic at all levels of investigation.

Young's modulus for the finite element analysis was arbitrarily chosen as $E=1$, the Poisson ratiov was chosen as $v$ $=0.25$, and the applied compressive stress $p$ was chosen as $p=1$. The $x, y$ coordinate system in Fig. 1 has its origin at the crack tip, i.e., $x_{o}=y_{o}=0$.

The numerical results of our analysis are plotted in Figs. 5 and 6. The inset of Fig. 5(a) shows the curved crack approximated by straight segments. The outer part of the crack intersects the free surface with an angle of $\varphi\left(\varphi=90^{\circ}\right.$ corre- 

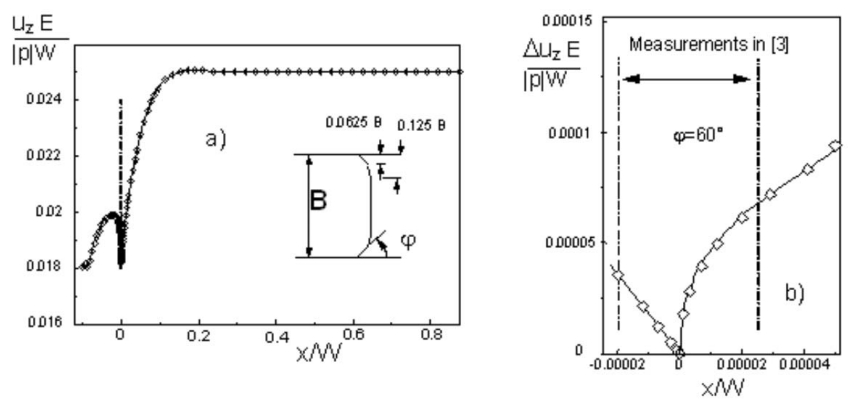

FIG. 5. (a) $u_{z}$ displacements obtained for $\varphi=60^{\circ}$; (b) displacement very close to $x=0$.

sponds to a straight crack). The intermediate part of the crack was modeled as a straight line with an angle of $\left(\varphi+90^{\circ}\right) / 2$. In a short unpublished study, we found that the near-tip displacements $u_{z}$ were independent of changes made in the shape of the crack front in the inner regions of the specimen.

The $u_{z}$ displacements at the free surface are shown in Fig. 5(a) for an angle of $\varphi=60^{\circ}, y=0$. As the angle $\varphi$ increased [not shown in Fig. 5(a)], the same displacement behavior was found but at slightly greater distances from the tip [Fig. 5(a)]. In Fig. 5(b), the differences in displacements, $\Delta u_{z}$ $=u_{z}(x)-u_{z}(0)$, are plotted for distances from the tip that are comparable to the region of measurement in Ref. 3.

Figure 6 illustrates the differences in displacements along the $y$ axis, i.e., for $x=0$. The near-tip displacements of Figs. 5 (b) and 6 were fitted for small $(x, y)$ by a power law relationship according to Eq. (2), which is now in the following normalized form:

$$
u_{z} \frac{E}{|p| W}=A_{x, y}\left(\frac{(x, y)}{W}\right)^{\lambda},
$$

where $A_{x}$ results from a fit in the $x$ direction and $A_{y}$ results from that in the $y$ direction. The exponent $\lambda$ numerically

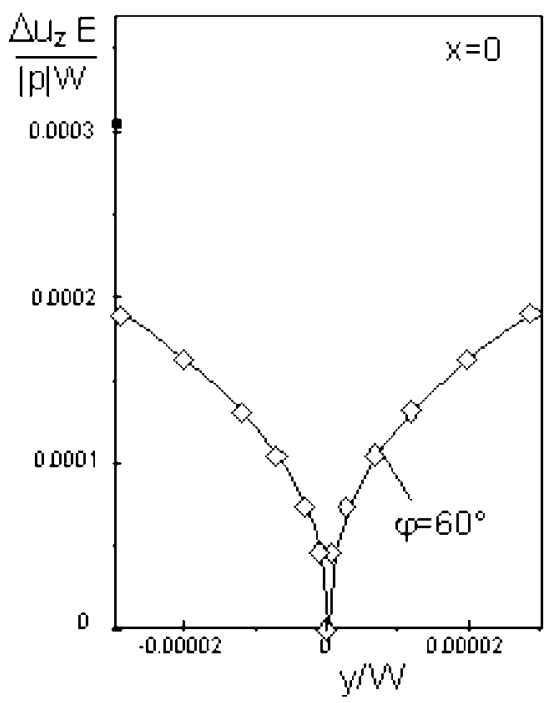

FIG. 6. Representation of the differences in displacements, $\Delta u_{z}=u_{z}(y)-u_{z}(0)$, along $x=0$ in the region of measurement obtained for $\varphi=60^{\circ}$.
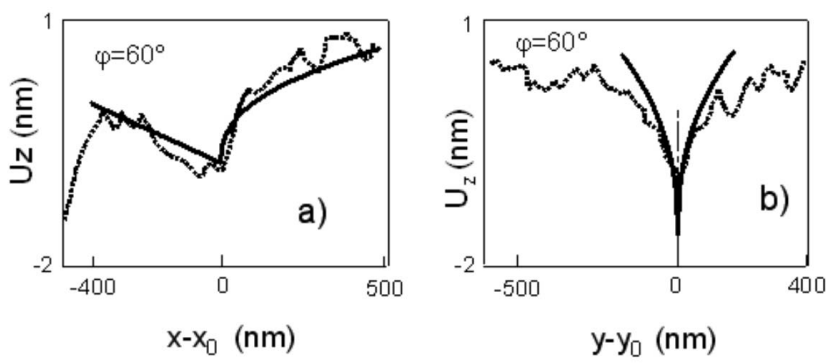

FIG. 7. Comparison of the finite element results for $\varphi=60^{\circ}$ (solid curves) with the measured surface displacements from Ref. 3 for $K=0.43 \mathrm{MPa} \sqrt{m}$ : (a) line $y=0$; (b) line $x=x_{0}$.

found from the fitting procedure was $\lambda=0.41$ for $x=0$ and $\lambda=0.43$ for $y=0$, which are in excellent agreement with the theoretical result of $0.42 .^{22}$ This may be considered an indication of a sufficiently fine finite element mesh, one that is comparable to the experimental results in Ref. 3.

The elastic displacements given in Eq. (3) are not singular. They are root shaped in the $+x$ and $\pm y$ directions since $\lambda>0$. In the wake of the crack $(y=0, x<0)$, the near-tip behavior is roughly linear, $u_{z} \sim-x$. From these results, we can conclude that the angular function of the root-shaped displacements disappears for $y=0, x<0$ or is at least significantly smaller than for the two other directions (i.e., $A_{-x}$ $\ll A_{x}$ ). Equation (3) is also continuous through the origin and hence satisfies the compatibility conditions.

\section{COMPARISON OF FINITE ELEMENT RESULTS WITH ATOMIC FORCE MICROSCOPY MEASUREMENTS}

The finite element results, which are summarized by the near-tip relationship in Eq. (3), can be used to predict the experimental displacements $u_{z}$. The stresses at the ends of the specimen and the crack length are not explicitly given in Ref. 3, but we are able to determine an effective compressive stress $p$ that causes $K_{I}=0.43 \mathrm{MPa} \sqrt{m}$, at which the experiments were performed. For the crack length of $c=2 \mathrm{~mm}$ $(c / R=4)$ used in our finite element model, this corresponds to $p \cong 70 \mathrm{MPa}$. The displacements computed for $2 \mathrm{~W}$ $=40 \mathrm{~mm}, E=70 \mathrm{GPa}$, and $\nu=0.25$ are shown in Figs. 7(a) and 7(b) as solid curves. Both the finite element analysis and the experiments from Ref. 3 show the same shape for the distribution of $u_{z}$ as a function of the distance from the crack tip. Since $0<\lambda<1$, the displacement profiles must exhibit a root-shaped $u_{z}$ dependency with an infinite steepness at the crack tip.

Referring now to Fig. 7, for small distances from the crack tip, good agreement between measurement and finite element computations was found, especially in Fig. 7(a), where the $\varphi=60^{\circ}$ curve follows the experimental curve over most of the graph, provided we ignore the waviness of the actual AFM data, which is due to surface roughness. To get a better comparison between the theoretical curves in Fig. 7 and the curves determined by AFM, the apparent surface noise due to roughness has to be added to the smooth theoretical curves in Fig. 7. This is done in the Appendix, where we show that when compared in this manner, the theoretical 


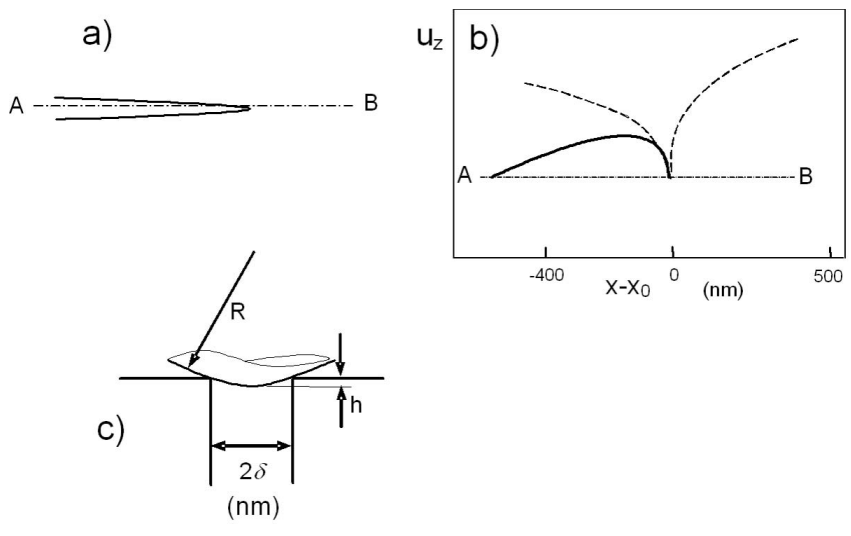

FIG. 8. Influence of the crack opening displacement on the measured surface displacement $u_{z}$.

and experimental curves show great similarity. Figure 7(b) only qualitatively follows the experimental data because the experimental curves seem to deviate from the finite element solution within the limits of the graph.

There is a significant difference in the trend of $u_{z}$ along $y=0$ for a distance of $x-x_{0}<-350 \mathrm{~nm}$ [Fig. 7(a)]. At first, the measured displacement increases in the same way as the computed displacements. Then, after about $350 \mathrm{~nm}$, the measured displacement rapidly decreases compared to the calculated displacement. In our opinion, this deviation represents the influence of an open crack. This is schematically illustrated in Fig. 8. For a scan along line $A B$ [Fig. 8(a)], the probe tip can enter the crack opening, which is to the left of the crack origin $\left(x_{o}, y_{o}\right)$, giving a superposition of true displacements $u_{z}$ and a contribution that arises from the crack opening. The open crack results in larger depth displacements since the sensor with a finite radius feels the open crack as a surface depression [Fig. 8(c)]. If $R$ denotes the sensor radius and $\delta$ is the crack opening displacement, the additional depth $h$ caused by the open crack for $\delta<R$ is

$$
h=R-\sqrt{R^{2}-\delta^{2}} \approx \frac{\delta^{2}}{2 R},
$$

with the approximate expression valid for $\delta \ll R$. In the neartip region, the crack opening displacement is given by

$$
\delta=C\left(x_{0}-x\right)^{\lambda}, \quad x<x_{0},
$$

as shown in Fig. 8(a). Combining Eqs. (4) and (5) gives an approximation for the measurable apparent depth displacements:

$$
u_{z, \text { measured }}=u_{z}(x)-\frac{C^{2}}{2 R^{2}}\left(x_{0}-x\right)^{2 \lambda} .
$$

For a scan along line $A B$ [Fig. 8(a)] for $x-x_{o}>0$, the correct surface displacements [dashed curve in Fig. 8(b)] are obtained if the scan probe stays on the specimen surface. If the probe enters into the crack, then an experimental scan along line $A B$ for $x-x_{o}<0$ gives a superposition of true displacements $u_{z}$ and a contribution that arises from the crack opening. The open crack results in larger depth displacements since the sensor with a finite radius feels the open crack as a deep surface depression [Fig. 8(c)].

\section{DISCUSSION}

In this section, we compare the two theoretical predictions of surface displacement with the AFM data presented in Ref. 3. Neither Eq. (1) nor the finite element analyses [Eq. (3)] are fits to the AFM data. They are both a priori calculations, which are based on theoretical considerations. Both predictions contain constants, such as Young's modulus and Poisson's ratio, that are physically based; neither contains an undetermined constant that has to be evaluated from the data. Therefore, both theories should be able to predict the displacement around the crack tip without further modification. We show that the predictions of Eq. (1) are about 4 orders of magnitude higher than the AFM measured displacements near the crack tip. By contrast, the prediction by the finite element analysis is only about $30 \%$ different from the measured displacement. The possible reasons for the difference in the case of the finite element analysis are discussed.

We also discuss the fitting procedure used in Ref. 3 to describe their AFM data. We note that the fitted curve has no basis in theory and its extrapolation beyond the region of the fit can have serious implications regarding the zero-point of the fit. Because minimization of the error to obtain the "best" fit of the curve to the data requires movement of the zeropoint of the fitted curve, the origin selected by the fit is not necessarily at the same location as the crack tip. In the case of Ref. 3, we suggest that the fitting procedure used by the authors place the origin of the fit into the wake of the crack, which is about $100 \mathrm{~nm}$ into the open crack, so that the displacement reported in Ref. 3 as a plastic zone was, in fact, the elastic depression on the wake side of the crack tip.

\section{A. Theoretical equations}

Equation (1) contains no free parameters that can be used in a minimization procedure to obtain a better fit of the equation to the data. All the quantities in Eq. (1) are either specimen dimensions or well-known physical constants. In order to check the equation against the AFM data, displacements have been computed for $\theta=0$ (along the $x$ axis), $r_{1}$ $=100 \mathrm{~nm}$ and $r_{2}=500 \mathrm{~nm}$. These distances are within the range of the measured displacements (Fig. 2). By introducing these geometrical data into Eq. (1) and using $B=4 \mathrm{~mm}, K_{I}$ $=0.43 \mathrm{MPa} \sqrt{m}, E=70 \mathrm{GPa}$, and $v=0.25$ as the test data, the $z$ displacements at the two locations are $u_{z 1}=-15.2 \mu \mathrm{m}$ for $r_{1}$ and $u_{z 2}=-6.8 \mu \mathrm{m}$ for $r_{2}$. The computed difference in the displacements between these two locations is $\delta u_{z}=u_{z 2}$ $-u_{z 1}=8400 \mathrm{~nm}$. For the same distance in the $y$ direction, one obtains $8400 \sqrt{2} \quad \mathrm{~nm} \approx 6000 \mathrm{~nm}$. The measured difference between $r_{1}$ and $r_{2}$ is $0.9 \mathrm{~nm}$ (as indicated by the difference between the two circles in Fig. 9). Thus, the prediction and measurement differ by a factor of approximately 9000, which is almost 4 orders of magnitude. These values are not shown in Fig. 9 because the scale of the calculated displacement is so much larger than the scale of the measured displacement. This result emphasizes the inadequacy of Eq. (1) as a predictor of the displacement in the vicinity of a crack tip. 


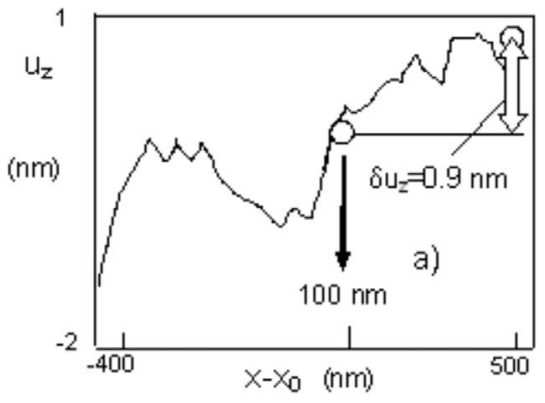

FIG. 9. Difference in displacements $\delta u_{z}$ between locations: $x$ $-x_{o}=100 \mathrm{~nm}$ and $x-x_{o}=500 \mathrm{~nm}$. The theoretical prediction based on Eq. (1) of $6000 \mathrm{~nm}$ is much larger than the measured displacement difference of $0.9 \mathrm{~nm}$.

Following a similar procedure for the finite element analysis along the crack plane with $\varphi=60^{\circ}[$ Fig. $7($ a) $]$ gives the difference, $\delta u_{z}=u_{z 2}-u_{z 1}=0.68 \mathrm{~nm}$, which is comparable to the measured difference of $0.9 \mathrm{~nm}$. For the surface displacements perpendicular to the crack plane [Fig. 7(b)], the calculated displacements are about $30 \%$ higher than the measured displacements. While not very large compared to the deviation calculated for Eq. (1), the deviations have a number of possible causes. First, the result presented in Fig. 1 represents just one scan of a surface containing an emerging crack. As the crack moves along, the AFM image of the crack will change its appearance from point to point, primarily as a consequence of surface roughness. If some "average" image of the depression could have been obtained, it is possible that the agreement of the finite element analysis with the AFM image would be even better than the estimate given here.

Surface roughness is important in the measurement of the surface depression. The rms roughness reported in Ref. 3 is $0.25 \mathrm{~nm}$, which means that $95 \%$ of the measured surface heights lie between $\pm 0.50 \mathrm{~nm}$. This height difference of about $1 \mathrm{~nm}$ is comparable to the magnitude of the depressions shown in Fig. 1 and will affect the final shape and magnitude of the crack-tip depression. The effects of roughness could be removed from the data shown in Figs. 2 and 3 by subtracting the original position of the specimen surface from the displaced position. Displacement data obtained in this manner should be independent of the surface roughness.

\section{B. Curve fitting}

\section{Least-squares procedure}

In Ref. 3, the authors fitted their experimental data with the following function: $u_{z}=-B / \sqrt{r}$. The functional dependence of the distance from the crack tip, $r$, was not justified in their paper, although it had the same functional dependence as Eq. (1). Interestingly enough, the equation fits the experimental data very well, passing right through the middle of the data. The authors used the fit to obtain the position of the crack tip, $x_{o}$, which required an extrapolation from the region of the fit to the point at which $u_{z}$ approaches negative infinity. The value of $x_{o}$ is then used to establish the width of the nonlinear zone, which is identified as a plastic zone in Ref. 3

The problem with the procedure used in Ref. 3 is that the fitting curve is extrapolated beyond the range of the data. This extrapolation procedure might be justified if the functional form used for the fit had theoretical support, but it does not. In fact, the fitting exponent used by the authors falls outside of the theoretically acceptable range for surface displacements surrounding an emerging crack, $\lambda=0-1 .{ }^{21,22}$ Because of this problem, one has to be cautious about using the value of $x_{o}$ established by this fit. Later in this paper, we will present an argument that the extrapolation used in Ref. 3 placed the origin approximately $100 \mathrm{~nm}$ behind the tip of the crack, which is almost exactly equal to the claimed size of the plastic zone in Ref. 3.

\section{General least-squares fit}

Before discussing the fit by Célarié et al. ${ }^{3}$ we perform a more general fit to the experimental data by using three fit parameters. The first is the crack-terminating angle, which is represented by the corresponding exponent $\lambda$ in the aluminosilicate glass. Instead of fixing the value of $\lambda$ at $-1 / 2$, as was done in Ref. $3, \lambda$ is now a variable of the fit. The second parameter is the exact location of the crack tip (i.e., the value of $x_{o}$ ). The third parameter is the minimum value of the displacements at $x=x_{o}$, i.e., $u_{z}\left(x_{o}\right)$.

Since the measurements for $y=0$ show a monotonically increasing curve with continuously decreasing steepness, it is possible to fit the measured data by arbitrary functions of the following type:

$$
u_{z}=C_{o}+C_{1}\left(x-x_{o}\right)^{n} \Rightarrow \Delta u_{z}=C_{1}\left(x-x_{o}\right)^{n}, \quad n \neq 0, \quad n<1
$$

with the location $x_{o}$ taken as a fit variable.

For the displacements along the $y$ axis, it is clear a priori by symmetry arguments where the minimum is. Therefore, the fit curve does not contain an unknown, $y_{o}$,

$$
u_{z}=D_{o}+D_{1} y^{n} \Rightarrow \Delta u_{z}=D_{1} y^{n}, \quad n \neq 0, \quad n<1 .
$$

Equations (7a) and (7b) include the theoretical solution with $n=\lambda$, as well as the assumption in Ref. 3 that $n=-1 / 2$. That exponent $n$, which simultaneously yields the best fit for two scanning lines, $y=0, x>x_{o}$ and $x=x_{o}$, was considered to be the solution to the fit problem.

The best exponent was found to be $n=\lambda=0.39$. This value corresponds to $\varphi=57^{\circ}$. The $x_{o}$ value found by this procedure is shifted by about $35 \mathrm{~nm}$ to the right of the fit in Ref. 3. This is already taken into account in Fig. 10(a). Figures 11(a) and 11(b) represent the same fit on a log-log plot. There is no indication of a plateau due to a plastic zone around the crack tip in Figs. 11(a) and 11(b).

Some general comments concerning the fit are in order. First, the constants of the least-squares fit are close to those used by the finite element analysis for which $\lambda=0.42$ and $\varphi=60^{\circ}$. The fit to the AFM data are better for the leastsquares fit than for the finite element analysis, which is expected since by definition, the error to the particular data set is minimized to obtain the best fit. Does this mean that the 

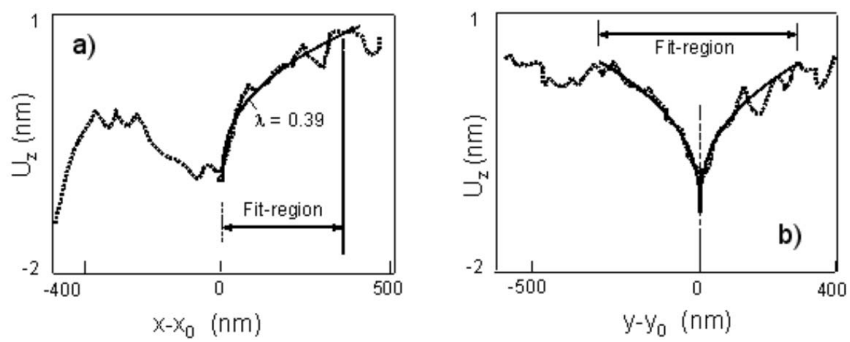

FIG. 10. Fitting curves for $u_{z}$ : (a) fit according to Eq. (7a) along $y=0$; (b) fit by Eq. (7b) along $x=x_{0}$.

least-squares fit is better than the finite element analysis in expressing the depression around a crack tip? Not necessarily, since the fit given here is only for a single set of AFM data. As the crack moves, the AFM image will change. If one considers scatter in the AFM image due to movement of the crack and surface roughness, the finite element analysis probably gives a better description of the mean surface displacement than the least-squares fit of a single image.

\section{Application of the least-squares fit to the finite element analysis results}

In this final section, we directly compare the finite ele-

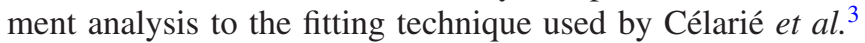
to obtain the zero position of the crack tip. We will assume that the finite element analysis provides a correct, unbiased description of a depression caused by an emerging crack in a homogeneous, isotropic material, such as a commercial glass. In the finite element analysis, the position of the origin and the shape of the depressed area are known and characterized. We then perform a least-squares fit of the equation $u_{z}=B / \sqrt{r}$ (Ref. 3) to the surface displacements obtained from the finite element analysis. Following the same procedure as used in Ref. 3, only the displacements to the right of the crack origin in Fig. 7(a) are used in the least-squares fit analysis.

The results of this calculation are shown in Fig. 12. The profile of the depressed area around the crack tip from the finite element analysis is given by the solid line to the left of the origin and by the solid curve to the right; it is the same profile shown in Fig. 7(a). The + symbols are the points on
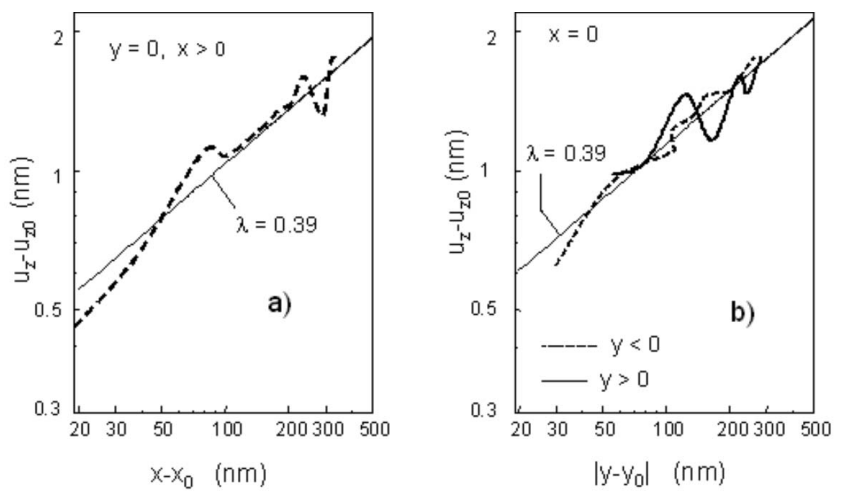

FIG. 11. These curves represent the same set of data as in Fig. 10 but in a logarithmic format.

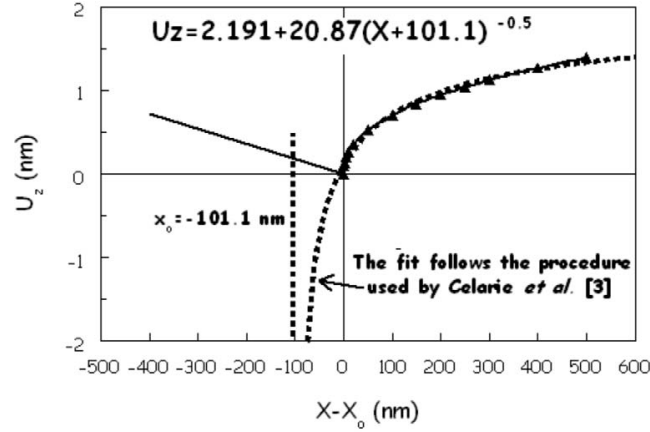

FIG. 12. A comparison of the finite element analysis results with the technique used in Ref. 3. The crack is advancing from the left to the right. Note that $x_{o}$ for the least-squares fit lies $101.1 \mathrm{~nm}$ to the wake side of the advancing crack. The equation gives the displacement as a function of $x$.

the finite element curve that were used for the least-squares fit. The least-squares fit (assuming $u_{z} \sim 1 / \sqrt{r}$ ) is given by the dashed curve in Fig. 12.

First, we note that the dashed curve and the solid curve are almost congruent within the data set. The main difference between the two curves is the location of the zero points. The zero point for the finite element analysis is at zero (by definition). The zero point for the least-squares fit lies $101.1 \mathrm{~nm}$ to the left of the crack origin given by the finite element analysis. Therefore, the crack origin, which is estimated from the least-squares fit, actually lies in the wake of the crack. Any measured displacement in this wake represents the elastic depression of the specimen surface behind the crack tip. It does not represent the plastic deformation near the crack tip, as was suggested in Ref. 3. The interpretation in Ref. 3 occurs because the functionality of the fitted curve is incorrect. Fitting the curve to the data requires an extrapolation of the curve beyond the region of the data, resulting in a crack-tip position that is off by about $100 \mathrm{~nm}$.

\section{SUMMARY}

Three-dimensional finite element computations were carried out for DCDC specimens made of glass. The surface displacements $u_{z}$ were computed for the free surface near a crack tip. The analysis was performed under the assumption of a pure linear-elastic material behavior. The comparison between our calculations and experimental results from literature was consistent with the hypothesis that the material in the vicinity of crack tips in silicate glasses behaves in a linear-elastic fashion. The results are not consistent with the premise that the crack-tip material behaves in a nonlinear or "plastic" fashion.

We also explored the consequence of fitting the AFM data from Ref. 3 with power law functions. Asymptotic analysis solutions for displacements in the vicinity of the crackterminating point have the form $u_{z} \sim r^{\lambda}$, where $\lambda \geq 0$. The finite element analysis yields values of $\lambda$ that are a function of the crack-terminating angle $\varphi$ and fit within the acceptable range for $\lambda$. Along the projected crack plane, $\theta=0$, the value 
of $\lambda=0.42$ for $\varphi=60^{\circ}$ is obtained from the finite element analysis. If a general power law function is fitted to the AFM displacement data, a value of 0.39 is obtained for $\lambda$, which is close to the value obtained from the finite element analysis. The value of $\lambda=0.39$, in turn, yields a value of $57^{\circ}$ for the angle $\varphi$, an angle that is similar to the value of $60^{\circ}$ measured on a soda lime silicate glass.

If $\lambda$ is forced to take on a value of -0.5 , as in Ref. 3 , and is fitted to the displacements from the finite element analysis, $\theta=0$, the resulting equation fits the displacements very well within the range of the data; however, in projecting the equation out of the range of the data, we find that the crack origin $x_{o}$ now lags about $100 \mathrm{~nm}$ behind the actual crack origin, as defined by the finite element calculation. We suspect that the same thing happened in Ref. 3. A consequence of this procedure is that the displacements measured by the AFM within the region behind the crack tip represent not a plastic zone but the elastic displacements on the trailing edge of the crack.

\section{ACKNOWLEDGMENTS}

The authors would like to thank B. R. Lawn and E. Fuller, Jr. (NIST) for their helpful discussions and review of this paper.

\section{APPENDIX}

As noted in Sec. IV, the data resulting from the AFM surface scan do not exactly correspond to the expected theoretical curve. There are two effects that contribute to this difference. First, the surface is never perfectly flat, so residual relief in the surface intersected by the crack front will affect the shape of the depressed area around the crack tip. Second, electronic noise from the microscope always yields a slight jitter to the image, which is noticeable at high resolutions. The combined result of these effects is an effective roughness of the surface, which is superimposed on the theoretical displacement caused by the stress field of the crack. The sum of these two effects (apparent surface roughness and displacement caused by the crack tip) is what the AFM detects.

When comparing experimental and theoretical results, the effect of this surface roughness has to be taken into account to see whether the theory is in agreement with the observations. Thus, for the theory laid out on the present work, a set of simulations was carried out to determine what the acquired AFM images should look like in practice.

The exact procedure used is as follows. First, the rms values for the original surface and the electronic noise are estimated. For each of these values, a noise set with the corresponding rms value as its standard deviation is generated. The chosen method for noise generation was successive random addition with $H=0.5$ as the roughness exponent. ${ }^{25}$ Each noise set is equivalent to a nonpersistent Brownian random walk.

The base line of each generated noise set, which is calculated by first order linear regression, is then subtracted from

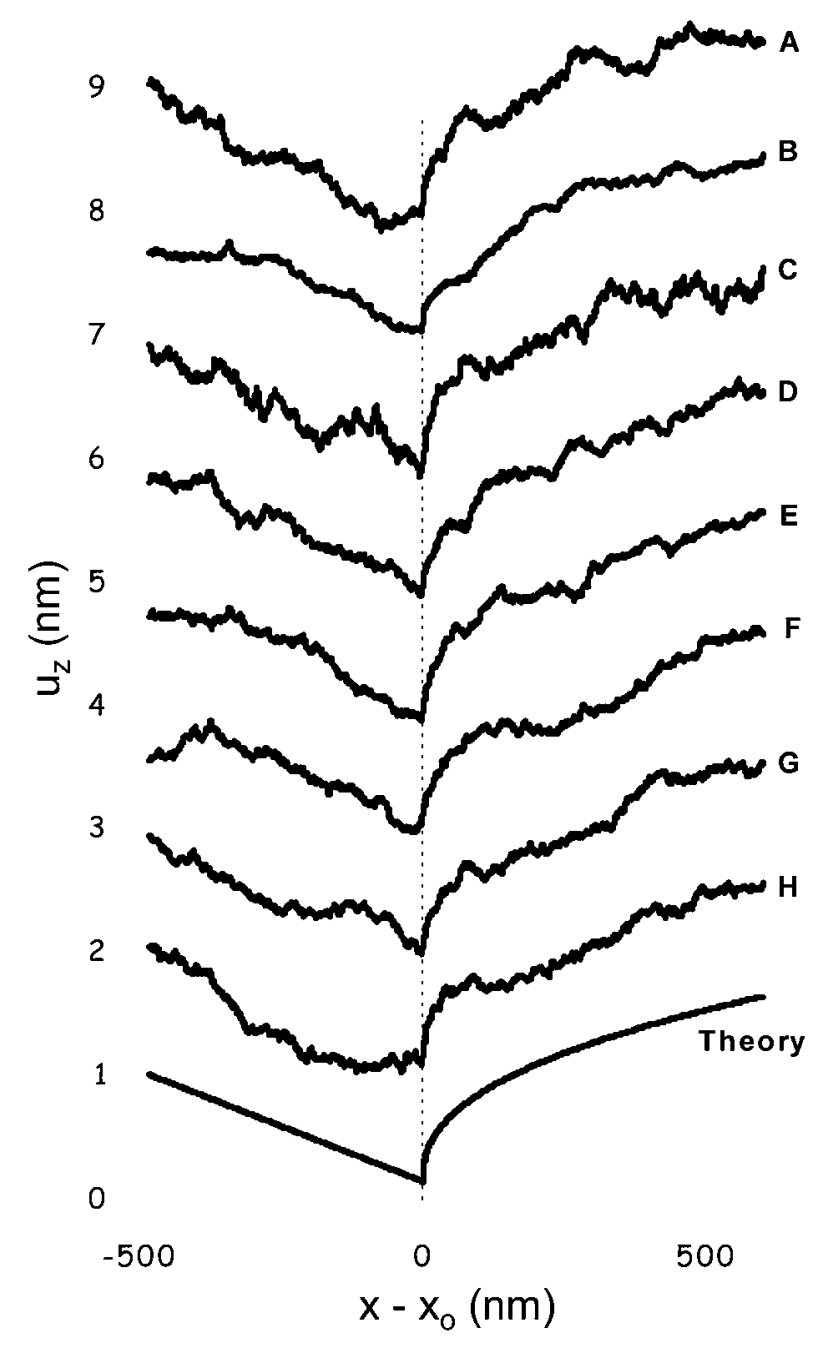

FIG. 13. Superposition of noise on the theoretical function for the surface displacement near a crack tip penetrating through a free surface. The theoretical curve is for a crack making an angle of $60^{\circ}$ with the external surface. The tip of the theoretical curve is indicated by the dotted line.

it. This procedure mitigates the influence of long wavelengths in the generated noise and removes any dc (constant) component on it. This is expected for a polished surface (polishing removes long-range, long-amplitude waviness from the surface), as well as a very common step in AFM data processing, usually referred to as "flattening" of the data. The rms value of the resulting sets can be adjusted by scaling each of them by an appropriate constant.

The "surface" noise is superimposed on the theoretical curve [Fig. 7(a)] to approximate the real surface profiles. The data are then convoluted with the tip shape (a spherical tip was assumed), so that the effects of the finite size of the tip are taken into account. Finally, the "electronic" set of noise from the AFM is added. The result is what the surface is expected to look like when scanned with an AFM if the underlying theory is correct.

Based on what is known of the data from Ref. 3, the tip radius was assumed to be $10 \mathrm{~nm}$, and the final rms value of the roughness was assumed to be about $0.1 \mathrm{~nm}$. This rough- 
ness was assumed to contain both the noise due to surface roughness and the electronic noise of the AFM. Both of these are usually contributors to the measured surface roughness when using an AFM to measure roughness. It is worth noting that, for the chosen range of observation (about $1 \mu \mathrm{m}$ ) and tip size, the influence of the tip shape was found to be negligible. In practical terms, this means that it is unnecessary to distinguish between the two rms values (surface and electronic) as long as the total rms value is kept constant.
The results of eight representative simulations are summarized in Fig. 13, which are in the curves labeled $A-H$. The original theoretical curve is also included for comparison. A vertical guide line is drawn for $x=x_{o}$, which helps identify where the origin is in the simulated data. The similarity between these results and the original experimental data in Fig. 2 is evident. We therefore conclude that the simulations are in good agreement with the experimental results.
${ }^{1}$ E. Guilloteau, H. Charrue, and F. Creuzet, Europhys. Lett. 34, 549 (1996).

${ }^{2}$ S. Hénaux and F. Creuzet, J. Am. Ceram. Soc. 83, 415 (2000).

${ }^{3}$ F. Célarié, S. Prades, D. Bonamy, L. Ferrero, E. Bouchaud, C. Guillot, and C. Marlière, Phys. Rev. Lett. 90, 075504 (2003).

${ }^{4}$ B. R. Lawn, B. J. Hockey, and S. M. Wiederhorn, J. Mater. Sci. 15, 1207 (1980).

${ }^{5}$ A. Kelly, W. R. Tyson, and A. H. Cotterell, Philos. Mag. 15, 567 (1967).

${ }^{6}$ S. M. Wiederhorn, B. J. Hockey, and D. E. Roberts, Philos. Mag. 28, 783 (1973).

${ }^{7}$ B. J. Hockey and B. R. Lawn, J. Mater. Sci. 10, 1275 (1975).

${ }^{8}$ H. Tanaka, Y. Bando, Y. Inomata, and M. Mitomo, J. Am. Ceram. Soc. 71, C32 (1988).

${ }^{9}$ H. Tanaka and Y. Bando, J. Am. Ceram. Soc. 73, 761 (1990).

${ }^{10}$ D. R. Clarke and K. T. Faber, J. Phys. Chem. Solids 48, 1115 (1987).

${ }^{11}$ J. P. Guin and S. M. Wiederhorn, Phys. Rev. Lett. 92, 215502 (2004).

${ }^{12}$ J. P. Guin and S. M. Wiederhorn, Int. J. Fract. 140, 15 (2006).

${ }^{13}$ J. P. Guin and S. M. Wiederhorn, Ceram. Trans. 199, 13 (2007).

${ }^{14} \mathrm{Ch}$. Janssen, Proceedings of the Tenth International Congress on Glass (Ceramic Society of Japan, Tokyo, Japan, 1974), Vol. 10, pp. 23-30.

${ }^{15}$ T. A. Michalske, Eng. Fract. Mech. 45, 637 (1993).

${ }^{16}$ M. Y. He, M. R. Turner, and A. G. Evans, Acta Metall. Mater. 43, 3453 (1995).

${ }^{17}$ G. C. Sih, Int. J. Fract. Mech. 7, 39 (1971).

${ }^{18}$ J. P. Benthem, Int. J. Solids Struct. 13, 479 (1977).

${ }^{19}$ Z. P. Bazant and L. F. Estenssoro, Int. J. Solids Struct. 15, 405 (1979).

${ }^{20}$ D. Leguillon and E. Sanchez-Palencia, Computation of Singular Solutions in Elliptic Problems and Elasticity (Wiley, Paris, 1987).

${ }^{21}$ A. Dimitrov, F.-G. Buchholz, and E. Schnack, Proceedings of the Fifth World Congress in Computational Mechanics (WCCMV), Vienna, Austria, 7-12 July 2002, edited by H. A. Mang, F. G. Rammerstorfer, and J. Eberhardsteiner (http://wccm.tuwien.ac.at/index.html).

${ }^{22}$ A. Dimitrov, F.-G. Buchholz, and E. Schnack, Science 12, 1 (2006).

${ }^{23}$ D. Leguillon and E. Sanchez-Palencia, Int. J. Fract. 99, 25 (1999).

${ }^{24}$ A. Dimitrov, Ph.D. thesis, University of Karlsruhe, 2002.

${ }^{25}$ J. Feder, Fractals (Plenum, New York, 1988). 